\title{
A Systematic Formulation of Slow Polaritons in Atomic Gases
}

\author{
G. Juzeliūnas ${ }^{1,2}$ and H. J. Carmichael ${ }^{2}$ \\ ${ }^{1}$ Institute of Theoretical Physics and Astronomy, A. Goštauto 12, Vilnius 2600, Lithuania \\ ${ }^{2}$ Department of Physics, University of Oregon, Eugene, OR 97403-1274, USA
}

(October 30, 2018)

We formulate a theory of slow polaritons in atomic gases and apply it to the slowing down, storing, and redirecting of laser pulses in an EIT medium. The normal modes of the coupled matter and radiation are determined through a full diagonalization of the dissipationless Hamiltonian. Away from the EIT resonance where the polaritons acquire an excitedstate contribution, lifetimes are introduced as a secondary step. With detuning included various four-wave mixing possibilities are analyzed. We investigate specifically the possibility of reverting a stopped polariton by reversing the control beam.

PACS numbers: 42.50.Gy, 32.70.Jz, 42.50.Fx, 03.75.Fi

Recently, electromagnetically induced transparency (EIT) 11 四 was shown to slow down dramatically [5], or even to stop completely [6,7], laser pulses in atomic gases. The experiments involve media of three-level atoms interacting with two lasers - a control beam and a probe pulse. The atoms have two hyperfine ground states, $|g\rangle$ and $|q\rangle$, and an electronically excited state $|e\rangle$, as illustrated in Fig. 1(a). Level $g$ is populated initially, before applying the probe pulse to couple $g$ and $e$. The role of the control beam is to introduce a transparency window so that the probe pulse propagates slowly in the medium.

Such behavior can be understood in terms of a branch of slow polaritons appearing between two close atomic resonances, as considered by Juzeliūnas (see Fig. 2(b) in [8]). Indeed, the control laser couples the states $|q\rangle$ and $|e\rangle$ dynamically, bringing level $q$ into resonance with the excited level $e$. The excited level splits, then, into the doublet shown in Fig. 1(b), giving precisely the level structure required to form a branch of slow polaritons. (a)

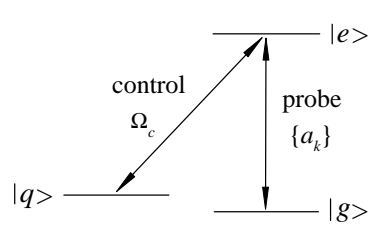

(b)

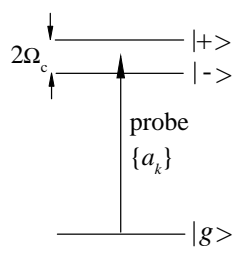

FIG. 1. (a) Atomic level scheme in the $\Lambda$ configuration of EIT. (b) Equivalent scheme incorporating the dynamical coupling of $q$ and $e$ characterized by the Rabi frequency $\Omega_{c}$.

Polaritons are the normal modes of a combined system of radiation and matter and are a familiar subject in solid state physics. Over the last decade the polariton idea has been applied widely to describe the quantized radiation field in dielectric media [8 14. Most studies, however, considered media of two-level atoms, and hence cannot accommodate the slow EIT polaritons. Slow polaritons appear in the analysis beyond two levels [8,13,14]; although the existing theoretical work does not deal with EIT, specifically. EIT (dark state) polaritons were first considered theoretically by Mazets and Matisov [15], and later by Fleischhauer and Lukin [16,17] who suggested storing the probe pulse (stopping the polariton) by adiabatically switching off the control laser.

In this paper we present a systematic description of slow polaritons in EIT media. The theory is developed for atomic Bose-Einstein condensates (BECs), but is also applicable to ordinary atomic gases. In contrast to previous work 15 17], we explicitly diagonalize the full Hamiltonian, including detuning from the EIT resonance and the contact interaction, and without making the rotating wave approximation in the interaction with the probe field. Away from the EIT resonance the polaritons acquire an excited-state contribution which leads to a finite radiative lifetime. With detuning included various fourwave mixing possibilities are suggested and we specifically propose a scheme for reverting a stopped polariton. We also apply our formulation to the recent experiments [6,7] where it is apparent from the explicitly constructed polariton modes that the storage and retrieval of the probe pulse takes place at the medium boundaries (to within an extremely small correction) and stopping the polariton can be achieved even by a sudden "turn-off" of the control laser.

Consider a gas of Bose atoms with an internal energy level structure as depicted in Fig. 1(a). The atoms populate the state $|g\rangle$ and form a Bose-Einstein condensate characterized by the field-operator $\widehat{\psi}_{g}(\mathbf{r})=\rho_{0}^{1 / 2}+$ $\Delta \widehat{\psi}_{g}(\mathbf{r})$, where $\rho_{0}=N_{0} / V_{0}$ is the density of the (homogeneous) condensate 18 and the field operator $\Delta \widehat{\psi}_{g}(\mathbf{r})$ describes small deviations from an ideal condensate due to non-condensate atoms. The Bose field-operators that account for the other two electronic states are expanded in terms of plane waves as

$$
\widehat{\psi}_{j}(\mathbf{r})=V_{0}^{-1 / 2} \sum_{\mathbf{k}} b_{j, \mathbf{k}} e^{i \mathbf{k} \cdot \mathbf{r}}
$$

where $b_{\mathbf{k}, j}$ annihilates an atom with internal state $|j\rangle$ $(j=q, e)$ and wave-vector $\mathbf{k}$. A classical control field, 
with wave-vector $\mathbf{k}_{c}$ and angular frequency $\omega_{c}$, couples states $|q\rangle$ and $|e\rangle$. In the presence of this field, the Hamiltonian for the excited atoms reads

$$
\begin{array}{r}
H_{\mathrm{atom}}=\hbar \sum_{\mathbf{k}}\left[\omega_{e, \mathbf{k}} b_{e, \mathbf{k}}^{\dagger} b_{e, \mathbf{k}}+\left(\omega_{q, \mathbf{k}-\mathbf{k}_{c}}+\omega_{c}\right) b_{q, \mathbf{k}}^{\dagger} b_{q, \mathbf{k}}\right. \\
+\Omega_{c} b_{q, \mathbf{k}-\mathbf{k}_{c}}^{\dagger} b_{e, \mathbf{k}}+\Omega_{c} b_{e, \mathbf{k}}^{\dagger} b_{\left.q, \mathbf{k}-\mathbf{k}_{c}\right]}
\end{array}
$$

where $\omega_{j, \mathbf{k}}=\omega_{j}+\hbar k^{2} / 2 M(j=q, e)$ are the atomic excitation energies (in units of $\hbar$ ), and $\Omega_{c}$ is the Rabi frequency determining the magnitude of the excited-state splitting. To remove an explicit time dependence due to the control field we have adopted a rotating frame (frequency $\omega_{c}$ ) for the state $|q\rangle$.

Adding now the interaction with a quantized probe field, the Hamiltonian for the combined system of radiation and matter is written

$$
H=H_{\text {atom }}+H_{\text {rad }}+H_{\text {rad-atom }}+H_{\text {cont }} .
$$

Here $H_{\mathrm{rad}}$ is the radiative Hamiltonian,

$$
H_{\mathrm{rad}}=\sum_{\mathbf{k}} \hbar c k\left(a_{\mathbf{k}}^{\dagger} a_{\mathbf{k}}+1 / 2\right),
$$

where $a_{\mathbf{k}}$ is the annihilation operator for a probe photon (polarized along the dipole moment $\mu$ of the $g \rightarrow e$ transition), and the summation is over all wave-vectors, $\mathbf{k} \equiv \mathbf{k}_{p}$, in the probe pulse wave-packet; the operator $H_{\text {rad-atom }}=-\varepsilon_{0}^{-1} \int \mathbf{d}(\mathbf{r}) \mathbf{p}(\mathbf{r}) d^{3} \mathbf{r}$ describes the interaction between the probe field and the atoms, where $\mathbf{d}(\mathbf{r})$ and $\mathbf{p}(\mathbf{r})=\mu \widehat{\psi}_{e}(\mathbf{r})^{\dagger} \widehat{\psi}_{g}(\mathbf{r})+$ h.c. are the electric displacement and polarization field operators, respectively; the last term, $H_{\text {cont }}=\left(x / 2 \varepsilon_{0}\right) \int \mathbf{p}(\mathbf{r}) \mathbf{p}(\mathbf{r}) d^{3} \mathbf{r}$, represents the contact interaction which appears in the multipolar formulation of QED [19 22], commonly adopted with $x=1$. We choose $x=2 / 3$ in the contact interaction [23]. This yields the correct local field corrections in the refractive index [Eq. (8)].

As a first step we take into account only coherent optical processes in which the absorption of a photon promotes a condensate atom to the excited electronic state from which it returns, via photon emission, to the condensate. For this purpose, we make the replacement $\widehat{\psi}_{g}(\mathbf{r}) \rightarrow \rho_{0}^{1 / 2}$ in the polarization field $\mathbf{p}(\mathbf{r})$. In doing this, incoherent processes, such as spontaneous emission to modes other than that of the absorbed photon, are disregarded. Spontaneous emission will be analyzed in a second step later on. With the replacement, one has

$$
H_{\mathrm{rad}-\text { atom }}=-i \sum_{\mathbf{k}} \mu \sqrt{\frac{\hbar c k \rho}{2 \varepsilon_{0}}}\left(a_{-\mathbf{k}}-a_{\mathbf{k}}^{\dagger}\right)\left(b_{e, \mathbf{k}}+b_{e,-\mathbf{k}}^{\dagger}\right)
$$

and

$$
H_{\mathrm{cont}}=x \sum_{\mathbf{k}} \frac{\mu^{2} \rho}{2 \varepsilon_{0}}\left(b_{e,-\mathbf{k}}+b_{e, \mathbf{k}}^{\dagger}\right)\left(b_{e, \mathbf{k}}+b_{e,-\mathbf{k}}^{\dagger}\right),
$$

where the transition dipole moment is chosen to be real.

The complete Hamiltonian, with the simplified terms (5) and (6), has been diagonalized by a Bogoliubov-type transformation, which mixes the radiation and matter modes to yield the polaritons. We thus obtain, adapting the methods of Juzeliūnas [8],

$$
H=\hbar \sum_{\mathbf{k}} \sum_{m=1}^{3} \omega_{\mathbf{k}}^{(m)} P_{m, \mathbf{k}}^{\dagger} P_{m, \mathbf{k}}+\text { const }
$$

where $m=1,2,3$ labels the polariton dispersion branches (Fig. 2) and the eigen-frequencies $\omega_{\mathbf{k}}^{(m)} \equiv \omega$ are to be determined from the equation $\omega=c k / n$, where $n \equiv n(\omega, \mathbf{k})$ is the refractive index, with

$$
n^{2}=\frac{1+x \alpha \rho / \varepsilon_{0}}{1-(1-x) \alpha \rho / \varepsilon_{0}} \quad(x=2 / 3)
$$

$\alpha$ is the atomic polarizability, given for $|\Delta \omega| \ll \omega$ by

$$
\alpha=-\frac{\mu^{2}}{\hbar} \frac{\Delta \omega}{\Delta \omega^{2}+\beta \Delta \omega-\Omega_{c}^{2}},
$$

where $\Delta \omega=\omega-\omega_{c}-\omega_{q, \mathbf{k}-\mathbf{k}_{c}}$ is the detuning from the two-photon resonance and $\beta=\omega_{q, \mathbf{k}-\mathbf{k}_{c}}+\omega_{c}-\omega_{e, \mathbf{k}}$ is the control laser frequency mismatch.
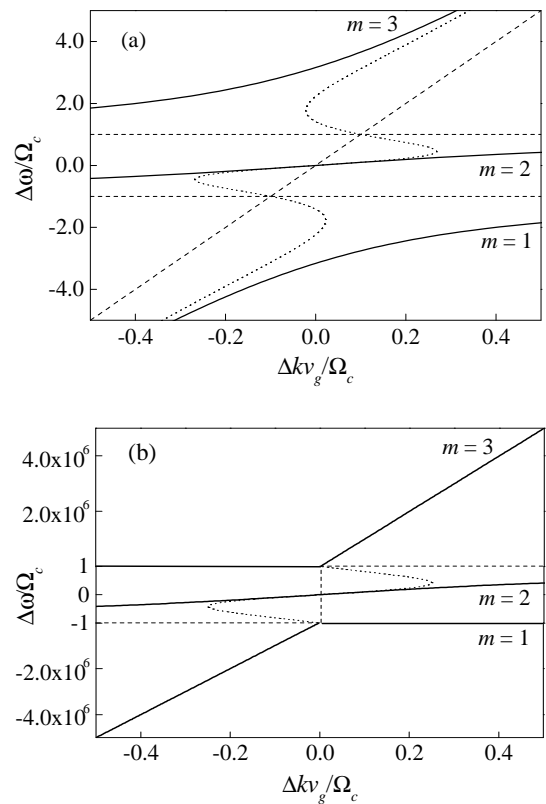

FIG. 2. Polariton dispersion branches (solid lines) in an EIT medium with $\beta=0$. The dashed lines show the uncoupled modes in the limit of vanishing density. The dotted curve is calculated from the semiclassical susceptibility and includes the excited state linewidth. The parameters in (b) are taken from the experiment of Liu et al. [6]; note the expanded frequency scale around the EIT resonance where the free-space photon branch is almost a vertical line. In (a) the density is reduced to give $v_{g} / c=10^{-1}$ so that the curves can be plotted using a single frequency scale. 
The polariton modes are defined by the Bose operators $P_{m, \mathbf{k}}$. These are determined explicitly to be $(|\Delta \omega| \ll \omega)$

$$
P_{m, \mathbf{k}}=P_{m, \mathbf{k}}^{(\mathrm{rad})}+i u b_{q, \mathbf{k}-\mathbf{k}_{c}}+i u\left(\Delta \omega / \Omega_{c}\right) b_{e, \mathbf{k}},
$$

where $u \equiv u_{m, \mathbf{k}}=\left(1-n v_{g} / c\right)^{1 / 2}\left[1+\left(\Delta \omega / \Omega_{c}\right)^{2}\right]^{-1 / 2}$ and

$$
P_{m, \mathbf{k}}^{(\mathrm{rad})}=\left(v_{g} / c\right)^{1 / 2}\left[(n+1) a_{\mathbf{k}}+(n-1) a_{-\mathbf{k}}^{\dagger}\right] / 2
$$

is the radiative component; here $v_{g}=c(n+\omega \partial n / \partial \omega)^{-1}$ is the radiative group velocity, and both $n \equiv n^{(m)}$ and $v_{g} \equiv v_{g}^{(m)}$ are to be calculated at $\omega=\omega_{\mathbf{k}}^{(m)}$.

In contrast to the polaritons considered in [15 17, the operator $P_{2, \mathbf{k}}$ represents an eigen-mode of the total Hamiltonian, not only at $\operatorname{EIT}$ resonance $(\Delta \omega=0)$, but for the entire branch of slow polaritons $(m=2)$. The systematic $\mathbf{k}$-space formulation we have presented facilitates a discussion of the frequency and wave-vector dependence of the radiation and matter fields.

The polariton dispersion branches are plotted in the vicinity of the EIT resonance in Fig. 2. Figure 2(b), in particular, adopts the parameters of the recent experiment, in which $v_{g} / c \approx 2 \Omega_{c}^{2} \hbar \varepsilon_{0} / \omega_{0} \mu^{2} \rho \approx 10^{-7}$ [6]. To aid comparisons with the EIT literature, where the refractive index is usually plotted, we also plot the dispersion curve (dotted line) given by the semiclassical susceptibility. The susceptibility includes the effect of the excited state lifetime and hence the dispersion curve continuously connects the three polariton branches.

In our theory the polaritons acquire a finite lifetime through the excited-state contribution to Eq. (10). The decay rate is $\Gamma=u^{2}\left(\Delta \omega / \Omega_{c}\right)^{2} \Gamma_{0}$, where $\Gamma_{0}$ is the rate of atomic radiative decay and $u \approx 1$ provided $|\Delta \omega| \ll \Omega_{c}$. Away from the EIT resonance, the polariton decays spontaneously at this rate into a photon and a translationally excited ground-state atom. In the experiment [6] the probe pulse excites a frequency band $|\Delta \omega| / \Omega_{c} \sim 0.02$. There is thus significant loss $(\sim 25 \%)$ due to polariton decay during the measured $11.8 \mu$ s propagation time in the medium. Some broadening of the probe pulse is expected from this as the decay rate is frequency dependent.

The contact interaction has no discernable effect on the slow polariton branch of Fig. 2. At the density currently used [6] its influence is at the level of less than $1 \%$. With a ten-fold increase in density, however, local field effects would no longer be negligible. The distinction between the radiative and full group velocity $\partial \omega / \partial k=\partial(\Delta \omega) / \partial k+\partial \omega_{q, k-k_{c}} / \partial k \approx v_{g}+\hbar\left(k-k_{c}\right) / M$ is also unimportant in current experiments. The atomic velocity, $\hbar\left(k-k_{c}\right) / M$, is much smaller than $v_{g}$ even for counter-propagating control and probe lasers (typically it is of the order of a few $\mathrm{cm} / \mathrm{s}$ ).

We consider now the time evolution of a wave-packet of EIT polaritons (a probe pulse in the medium) influenced by the following sudden perturbations: (i) at $t=t_{1}$ the original control beam is switched off, (ii) after some delay, at $t=t_{2}>t_{1}$, the control beam is again turned on with a new Rabi frequency $\Omega_{c}^{\prime}$ and wavevector $\mathbf{k}_{c}^{\prime}$. In contrast to what has been previously considered [6, 7, 16, 17], $\mathbf{k}_{c}^{\prime}$ does not necessarily coincide with the original wavevector $\mathbf{k}_{c}$. If $\mathbf{k}_{c}^{\prime}=-\mathbf{k}_{c}$, for instance, the regenerated probe pulse can move backwards, as we will show.

At $t<t_{1}$ the wave-packet is described by the statevector

$$
|t\rangle=\left|\left\{\alpha_{\mathbf{k}, t}\right\}\right\rangle \equiv \prod_{\mathbf{k}} \exp \left(\alpha_{\mathbf{k}, t} P_{2, \mathbf{k}}^{\dagger}-\text { h.c. }\right)|\operatorname{vac}\rangle,
$$

where $|v a c\rangle$ is the vacuum state-vector $\left(P_{2, \mathbf{k}}|\operatorname{vac}\rangle=0\right)$, $\left|\left\{\alpha_{\mathbf{k}, t}\right\}\right\rangle$ is a many-mode coherent state, and the amplitude $\alpha_{\mathbf{k}, t}=\alpha_{\mathbf{k}, t_{1}} \exp \left[-i \omega_{\mathbf{k}}^{(2)}\left(t-t_{1}\right)\right]$. At $t=t_{1}$ the control laser is suddenly switched off. Equation (12) then represents the initial condition for the subsequent time evolution, giving for $t_{1}<t<t_{2}$,

$$
|t\rangle=\prod_{\mathbf{k}} \exp \left(i u \alpha_{\mathbf{k}, t} b_{q, \mathbf{k}-\mathbf{k}_{c}}^{\dagger}-\text { h.c. }\right) \exp \left(S_{\mathbf{k}}\right)|\operatorname{vac}\rangle \text {. }
$$

The first exponent describes the magnetic excitations, which are now decoupled from the photons so that (up to a phase) $\alpha_{\mathbf{k}, t}=\alpha_{\mathbf{k}, t_{1}} \exp \left[-i \omega_{q, \mathbf{k}-\mathbf{k}_{c}}\left(t-t_{1}\right)\right]$. The operator $\exp \left(S_{\mathbf{k}}\right)$ accounts for the small radiative $\left(\sim 3 \times 10^{-4}\right)$ and excited state $(\sim 0.02)$ contributions to the slow polariton amplitude. These excitations are subsequently converted into spontaneously emitted photons and may be omitted for $t-t_{1}$ larger than the radiative lifetime. It should be emphasized that while the radiative contribution plays an essential role in giving the polariton its velocity while the control laser is "on," it accounts for a very small fraction of the quasiparticle number (when $\left.v_{g} / c \ll 1\right)$ and may simply be discarded to stop the polariton.

At $t=t_{2}$ the new control beam is applied. Expanding the magnetic operator $i b_{q, \mathbf{k}-\mathbf{k}_{c}}^{\dagger} \equiv i b_{q, \mathbf{k}^{\prime}-\mathbf{k}_{c}^{\prime}}^{\dagger}$ in terms of the new polariton operators $P_{m, \mathbf{k}^{\prime}}^{\prime \dagger}(m=1,2,3)$, the statevector evolves for $t>t_{2}$ as

$$
|t\rangle=\prod_{\mathbf{k}^{\prime}} \exp \left(u u^{\prime} \alpha_{\mathbf{k}^{\prime}, t}^{\prime} P_{2, \mathbf{k}^{\prime}}^{\prime \dagger}-\text { h.c. }\right) \exp \left(S_{\mathbf{k}^{\prime}}^{\prime}\right)|\operatorname{vac}\rangle
$$

with $\alpha_{\mathbf{k}^{\prime}, t}^{\prime}=\alpha_{\mathbf{k}, t_{2}} \exp \left[-i \omega_{\mathbf{k}^{\prime}}^{\prime(2)}\left(t-t_{2}\right)\right]$. The operator $S_{\mathbf{k}^{\prime}}$ accounts for the other polariton modes $(m=1,3)$ which are subsequently converted into spontaneously emitted photons. Here $\mathbf{k}^{\prime}=\mathbf{k}-\mathbf{k}_{c}+\mathbf{k}_{c}^{\prime}$ is the wave-vector of the regenerated polariton and the quantities $u^{\prime}, P_{2, \mathbf{k}^{\prime}}^{\prime}$, and $\omega_{\mathbf{k}^{\prime}}^{\prime(m)}$ are defined in the obvious way [24].

Such behavior may be viewed as a kind of time-delayed four-wave mixing (photon echo 25]) involving the original probe pulse, $\mathbf{k}$, two control beams, $\mathbf{k}_{c}$ and $\mathbf{k}_{c}^{\prime}$, and the regenerated probe pulse, $\mathbf{k}^{\prime}$, the wave-vectors satisfying the phase matching condition $\mathbf{k}-\mathbf{k}_{c}=\mathbf{k}^{\prime}-\mathbf{k}_{c}^{\prime}$. The distinctive feature is the involvement of slow EIT polaritons. Consequently very high conversion efficency 
can be reached $\left(u u^{\prime} \approx 1\right)$ if both the original pulse and the regenerated polaritons are in the vicinity of the EIT resonance. Note that there is no need for a smooth (adiabatic) "turn-off" and "turn-on" of the control laser to map the slow polariton into and out of a magnetic excitation. Even if the switching on and off are instantaneous, the reconstruction is almost perfect when $u u^{\prime} \approx 1$. The actual storage and retrieval of the probe pulse takes place at the medium boundaries where vacuum photons are converted into slow polaritons and vica versa.

We consider, finally, some specific situations. If the wavevector of the control laser does not change $\left(\mathbf{k}_{c}^{\prime}=\mathbf{k}_{c}\right)$, one arrives at degenerate four wave mixing in which the regenerated probe photon has the same wavevector as the original $\left(\mathbf{k}^{\prime}=\mathbf{k}\right)$. This is the situation investigated in recent work [6, [6, 16, 17]. On the other hand, with copropagating control and probe beams one can change the direction of the probe pulse by changing the direction of the control beam [26]. If $\mathbf{k}_{c}^{\prime}=-\mathbf{k}_{c}$ [27], then $\mathbf{k}^{\prime}=$ $-\mathbf{k}-2\left(\mathbf{k}_{c}-\mathbf{k}\right)$, and the reverted polariton experiences a frequency shift $\delta \omega^{\prime} \approx 2 v_{g}^{\prime}\left(k_{c}-k\right)$. Under the conditions of the recent experiment [6] one obtains $\delta \omega^{\prime} \approx 0.26 \mathrm{kHz} \times$ $2 \pi$, which is very small compared to the coupling Rabi frequency (and also the spectral width of the probe pulse) and the reverted polariton remains in the EIT region. For counter-propagating beams, the wave-vector of the new polariton is $\mathbf{k}^{\prime}=\mathbf{k}+2 \mathbf{k}_{c}^{\prime} \approx 3 \mathbf{k}$; the polariton becomes situated close to the upper band edge where there is a rate of radiative decay on the order of $\Gamma_{0}$ (unless $\Omega_{c}^{\prime}$ is extremely small) [28].

We have formulated a theory of slow polaritons in atomic gases and applied it to the slowing down, storing, and redirecting of laser pulses in an EIT medium. The polariton modes have been determined through a full diagonalization of the dissipationless Hamiltonian, and lifetimes introduced as a secondary step. With detuning included various four-wave mixing possibilities were analyzed. The possibility of reverting a stopped polariton was demonstrated.

The authors acknowledge helpful discussions with T. W. Mossberg, M. G. Raymer, M. Mašalas, and K. V. Krutitsky. Work supported by the NSF under Grant No. PHY-0099576 and by the Council for International Exchange of Scholars.

[1] E. Arimondo, In Progress in Optics, edited by E. Wolf (Elsevier, Amsterdam, 1996), p.257.

[2] S.E. Harris, Physics Today 50(7), 36 (1997).

[3] M.O. Scully and M.S. Zubairy, Quantum Optics (Cambridge University Press, Cambridge, 1997).

[4] K. Bergmann, T. Theuer and B.W. Shore, Rev. Mod.
Phys. 70, 1003 (1998).

[5] L.V. Hau, S.E. Harris, Z. Dutton, and C.H. Behroozi, Nature 397, 594 (1999).

[6] C. Liu, Z. Dutton, C.H. Behroozi and L.V. Hau, Nature 409, 490 (2001).

[7] D.F. Phillips, A. Fleischhauer, A. Mair, R.L. Walsworth and M.D. Lukin, Phys. Rev. Lett. 86, 783 (2001).

[8] G. Juzeliūnas, Phys. Rev. A 53, 3543 (1996).

[9] J. Knoester, and S. Mukamel, Phys. Rev. A 40, 7065 (1989).

[10] B. Svistunov and G. Schlyapnikov, Zh. Eksp. Theor. Phys. 98, 129 (1990) [Sov. Phys. JETP 71, 71 (1990)].

[11] H.D. Politzer, Phys. Rev. A 43, 6444 (1991).

[12] B. Huttner and S.M. Barnett, Phys. Rev. A 46, 4306 (1992); S.-T. Ho and P. Kumar, J. Opt. Soc. Am. B 10, 1620 (1993).

[13] P.D. Drummond and M. Hillery, Phys. Rev. A 59, 691 (1999).

[14] G. Juzeliūnas and D.L. Andrews, In Advances in Chemical Physics, v.112, edited by I. Prigogine and S. A. Rice (Wiley, New York, 2000), p.357.

[15] I. E. Mazets and B. G. Matisov, JETP Lett. 64, 515 (1996) [Pis'ma Zh. Exp. Theor. Fiz. 64, 473 (1996)].

[16] M. Fleischhauer and M.D. Lukin, Phys. Rev. Lett. 84, 5094 (2000).

[17] M. Fleischhauer and M.D. Lukin, quant-ph/0106066.

[18] The homogeneous model may also be suitable for the analysis of a trapped BEC in the local density (ThomasFermi) approximation, see G. Morigi and G.S. Agarwal, Phys. Rev. A 62, 013801 (2000).

[19] E.A. Power, Introductory Quantum Electrodynamics (Longmans, London, 1964).

[20] C. Cohen-Tannoudji, J. Dupont-Roc, and G. Grynberg, Photons and Atoms (Wiley, New York, 1989).

[21] M. Lewenstein, L. You, J. Cooper and K. Burnett, Phys. Rev. A 50, 2207 (1994).

[22] P. Meystre, Atom Optics (Springer, Berlin, 2001).

[23] In fact, by taking $x=1$, one implicitly assumes that the electrostatic interaction between atoms is given by $\left(2 \varepsilon_{0}\right)^{-1} \int \mathbf{p}^{\|}(\mathbf{r}) \mathbf{p}^{\|}(\mathbf{r}) d^{3} \mathbf{r}$, which contains the Dirac delta singularity at the origin characteristic of the dipole-dipole coupling between point atoms. This is an artifact because atoms, which are not point objects, do not overlap in reality. The singularity is eliminated by subtracting the term $\left(6 \varepsilon_{0}\right)^{-1} \int \mathbf{p}(\mathbf{r}) \mathbf{p}(\mathbf{r}) d^{3} \mathbf{r}$, leading to our $x=2 / 3$.

[24] The frequency $\omega_{c}^{\prime}$ might be tuned to another excited level $e^{\prime}$ leading to a substantial change in the frequency of the regenerated polariton.

[25] T.W. Mossberg, R. Kachru, S.R. Hartmann and A.M. Flusberg, Phys. Rev. A 20, 1976 (1979).

[26] This reversion of the probe pulse differs from that achieved by making the group velocity negative in a Doppler broadened gas: O. Kocharovskaya, Y. Rostovtsev and M.O. Scully, Phys. Rev. Lett. 86, 628 (2001).

[27] Under the conditions of the experiment [6], the circular polarization of the control laser should be reversed in order to drive the same transition $q \rightarrow e$.

[28] Such a polariton cannot leave the sample because it is far from the crossing point with the photon dispersion. Another reversion of the control laser would allow it to escape. 